\title{
Stroke Connectome and Its Implications for Cognitive and Behavioral Sequela of Stroke
}

\author{
Jae-Sung Lim, ${ }^{a}$ Dong-Wha Kang ${ }^{b}$ \\ ${ }^{a}$ Department of Neurology, Seoul Metropolitan Government-Seoul National University Boramae Medical Center, Seoul National University College of \\ Medicine, Seoul, Korea \\ bepartment of Neurology, Asan Medical Center, University of Ulsan College of Medicine, Seoul, Korea
}

Systems-based approaches to neuroscience, using network analysis and the human connectome, have been adopted by many researchers by virtue of recent progress in neuroimaging and computational technologies. Various neurological disorders have been evaluated from a network perspective, including stroke, Alzheimer's disease, Parkinson's disease, and traumatic brain injury. Until now, dynamic processes after stroke and during recovery were investigated through multimodal neuroimaging techniques. Many studies have shown disruptions in structural and functional connectivity, including in large-scale neural networks, in patients with stroke sequela such as motor weakness, aphasia, hemianopia, neglect, and general cognitive dysfunction. A connectome-based approach might shed light on the underlying mechanisms of stroke sequela and the recovery process, and could identify candidates for individualized rehabilitation programs. In this review, we briefly outline the basic concepts of structural and functional connectivity, and the connectome. Then, we explore current evidence regarding how stroke lesions cause changes in connectivity and network architecture parameters. Finally, the clinical implications of perspectives on the connectome are discussed in relation to the cognitive and behavioral sequela of stroke.

Keywords Connectome; Connectivity; Network; Stroke; Resting-state functional MRI; Diffusion tensor imaging

\author{
Correspondence: Dong-Wha Kang \\ Department of Neurology, Asan Medical \\ Center, University of Ulsan, College of \\ Medicine, 88 Olympic-ro 43-gil, \\ Songpa-gu, Seoul 05505, Korea \\ Tel: +82-2-3010-3440 \\ Fax: +82-2-474-4691 \\ E-mail: dwkang@amc.seoul.kr
}

Received: August 19, 2015

Revised: September 10, 2015

Accepted: September 17, 2015

This study was supported by National Research Foundation of Korea Grant NRF-2014R1A2A1A11051280 from the Korean government, and the Korea Health Technology R\&D Project, Ministry for Health \&t Welfare, Republic of Korea Grants $\mathrm{HI} 12 \mathrm{C} 1847$ and HI14C1983.

The authors have no financial conflicts of interest.

\section{Introduction}

\section{The need for a connectome-based approach}

The term "Connectome" was proposed by Olaf Sporns to denote "the complete set of all neural connections of the human brain.", Thereafter, this term has been widely used, and the human connectome project was initiated to investigate thorough and detailed connectivity maps of the human brain (http:// www.humanconnectomeproject.org).

Beyond the well-known concept of lesion-symptom mapping, recent studies have shown that specific cognitive and behavioral functions are not localized to anatomically restricted areas, but are rather routed to widespread neural networks, which are linked via several interconnected cortical areas. Localization of a specific symptom does not necessarily match the corresponding function of the brain region, particularly in the cortex. ${ }^{3}$ Furthermore, local destruction of certain anatomic regions can affect remote areas, even in the contralateral hemisphere, and lead to distributed dysfunction. ${ }^{4}$

Mechanisms for the symptoms in the acute phase of stroke and the dynamic changes throughout the recovery process during subacute to chronic phases of stroke remain to be deter- 
mined. What are the exact mechanisms by which lost functions are regained? What are the maladaptive or decompensatory mechanisms that lead to permanent deficits and post-stroke cognitive impairment? In particular, cognitive and behavioral sequela need to be approached from the perspective of systemsfailure, i.e. from a connectome perspective, considering their complex pathophysiology.

The human connectome has recently gained attention for its importance and possible implications for neuroscience as well as clinical neurology and psychology. Recent advances in neuroimaging and computational sciences have enabled the analysis of large and complicated data with relatively modest resources. These advances provide a general overview of the characteristics of the architecture and efficiency of the whole brain. In patients with stroke, alterations of these network characteristics might explain various intriguing symptoms and the recovery process, which are difficult to understand via classical lesionsymptom mapping.
In this review, we briefly outline the basic concepts of structural and functional connectivity, and connectome. Then, we explore current evidence regarding how stroke lesions cause changes in local and remote connectivities, large-scale neural networks, and general network architectures. Finally, the clinical implications of these connectome-based perspectives are discussed.

\section{Connectome and connectivity concepts}

\section{Networks and functional and structural connectivity}

Euler proposed graph theory to solve the Königsberg bridge problem in 1741 . $^{5}$ The concepts of network analysis and connectome are rooted in this theory. He has abstracted the problem by replacing landmasses of Königsberg map with 'nodes' and bridges with 'edges'. Based on this approach, Königsberg map was reconstructed to a mathematical graph. Recent neuroscience research has adopted this perspective to investigate various network attributes of brain disorders. ${ }^{6}$ By analogy, bridges
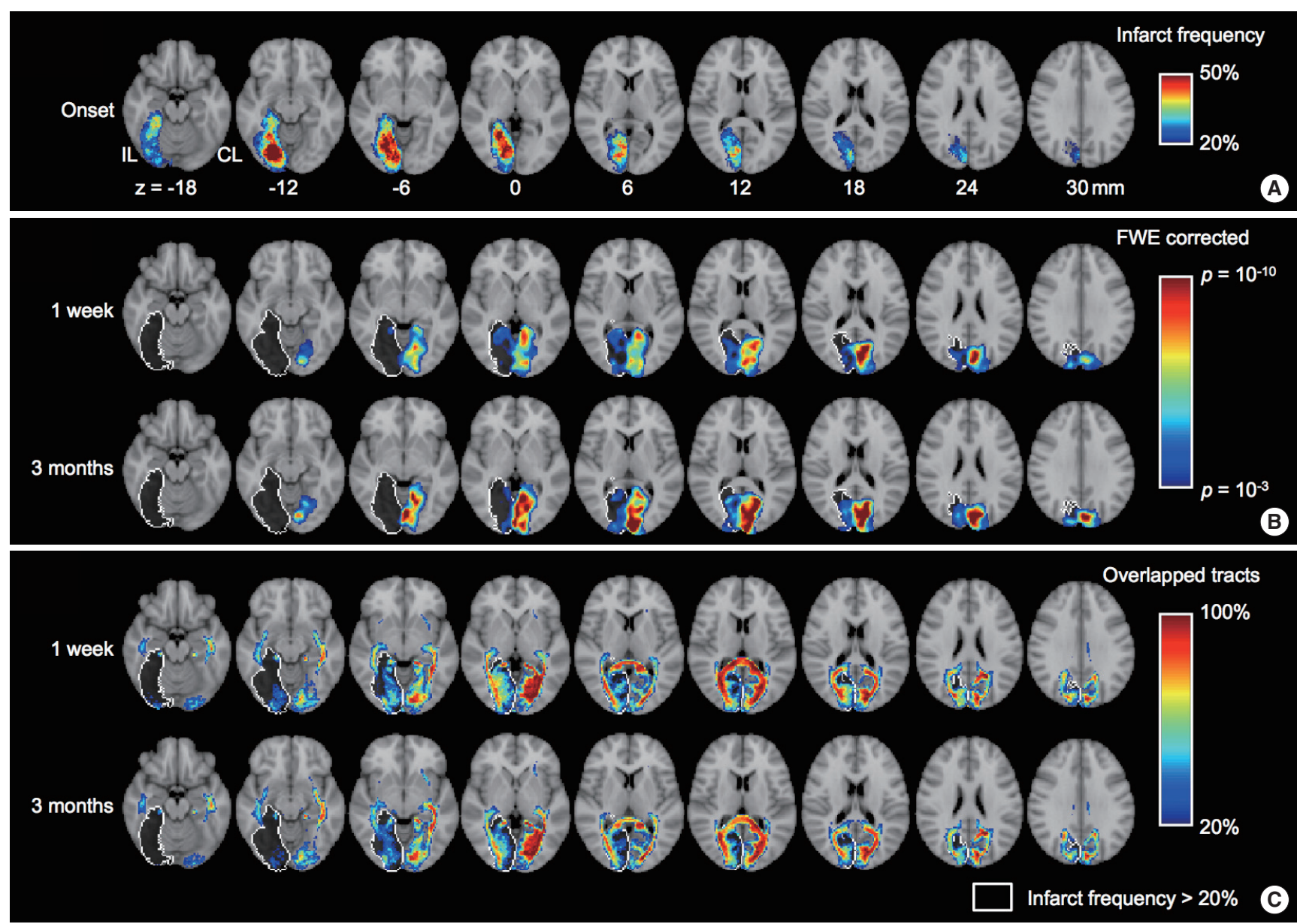

Figure 1. Alteration of functional and structural connectivity after stroke in the posterior cerebral artery territory $(\mathrm{n}=30)$. (A) Spatial pattern of infarct frequency across subjects. (B) Group level spatial patterns of visual network estimated by independent component analysis using resting-state fMRI. The one sample $t$ test was adopted to estimate the group level spatial patterns for each of time points (i.e., 1 week and 3 months from stroke onset). (C) Spatial pattern of overlapped probabilistic tracts connected with the visual cortex (regions-of-interest are calcarine, lingual and cuneus). The spatial patterns of functional and structural connectivity were superimposed over standard MNI T1 image. White colored outline indicates the infarct location which is greater than $20 \%$ of infarct frequency across subjects. 
correspond to the white matter tracts, and landmasses to the each cortical region.

There are two types of network analysis: functional connectivity and structural connectivity (Figure 1). Functional connectivity is defined by a temporal correlation in blood oxygen level-dependent signals in a resting-state or during specific tasks ${ }^{7}$ that can be measured by the time-series data of functional MRI (fMRI). Blood oxygen level-dependent contrast denotes signal differences in $\mathrm{T} 2{ }^{*}$-weighted images as a function of the amount of deoxygenated hemoglobin present. ${ }^{8}$ Brain activity consumes oxygen, decreasing the concentration of deoxygenated hemoglobin in neighboring cerebral vessels. This coupling of brain metabolism and blood flow is an underlying basic principle of fMRI. It enables visualization of changes in brain function over time using standard MRI scanners. Functional connectivity can also be investigated using electroencephalography and magnetic encephalography.

Task-related functional connectivity has been extensively studied in neuropsychology, and many specific human behav- ioral functions have been localized by these studies. However, study designs for task-related fMRI are generally complicated, and many stroke survivors cannot complete the tasks required in these experiments. Resting-state fMRI has the advantage that it does not require any specific tasks during imaging acquisition. ${ }^{9}$ Using resting-state fMRI analysis, evidence of extensive changes in large-scale neural networks (i.e., default mode network, central executive network, dorsal attention network, and salience network), have been reported for various disease states, and their possible roles in characterizing various neurological disorders have been suggested. ${ }^{10}$

Along with functional connectivity using resting-state fMRI, structural connectivity can be identified through diffusion tensor imaging and diffusion spectral imaging. The structural connections and fiber densities among predetermined cortical regions have been visualized, and these results are consistent with pre-existing anatomical knowledge of the major white matter tracts, such as the cingulum, uncinate fasciculus, arcuate fasciculus, and superior and inferior longitudinal fasciculi. These struc-
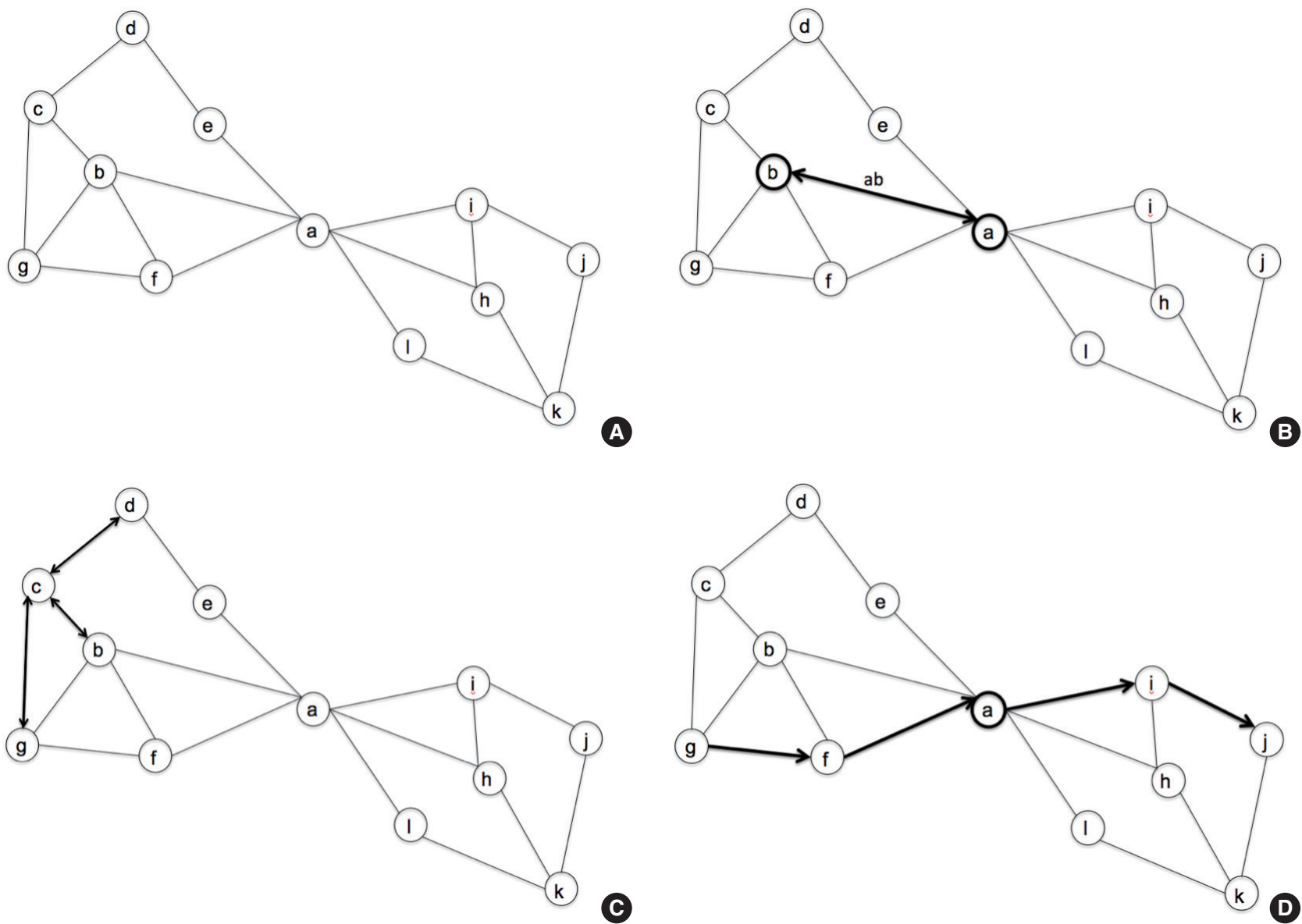

Figure 2. Representative diagram for the network characteristics. (A) This diagram consists of 12 nodes and their interconnections. (B) Node 'a' is interconnected with node ' $b$ ' through edge ' $a b$ '. (C) Degree of node ' $c$ ' is 3 , which denotes the edges ' $c d$ ', ' $b c$ ', and 'cg'. Likewise, the degree of node ' $b$ ' is 4 , and that of node ' $a$ ' is 6 . (D) Shortest path length between node ' $g$ ' and ' $j$ ' is 4 , which is visualized with arrows. 
tural connectivities have been suggested as useful in differentiating various neurodegenerative disorders. ${ }^{10}$

\section{Basic parameters for connectivity and connectome}

The basic elements of neural networks and connectome are nodes and edges. Nodes denote certain brain regions, which are usually predetermined cortical areas delineated by anatomical or functional classifications. An edge refers to the connection between two nodes, which could be anatomical or functional connections. ${ }^{11}$

Using the concepts of nodes and edges, some conceptual parameters have been proposed to reflect global network characteristics (Figure 2). 12 "Degree" means how many edges are connected to the corresponding node, and reflects the centrality of that node. For characterizing functional segregation of the network, a "module" consists of interconnected subnetworks, which might confer specific functions such as language or visuospatial attention. "Modularity" denotes the extent to which modules can describe the corresponding network. ${ }^{10}$ To measure network efficiency, the "shortest path length" reflects how many steps are required to move from a given node to another specific node. The "characteristic path length" is the average of all path lengths in the network, and is a measure of global connectivity. Finally, a "hub" is a node with a high degree that ensures network efficiency and integrity. ${ }^{10}$ More detailed explanations and the mathematical underpinnings of these parameters are well described in a position paper and a recent review. ${ }^{10,12}$

\section{Small-worldness of the network}

Based on these network parameters, the whole network may be classified as a regular network, random network, or smallworld network (Figure 3). ${ }^{10}$ The concept of small-world network was proposed by Duncan Watts and Stephen Strogatz, using rewiring probability in a ring lattice with a circular boundary. ${ }^{13}$ Classification is based on the characteristics of connections among neighboring and remote nodes. For example, in regular networks, the connections between neighboring nodes outweigh those between remote nodes. In random networks,
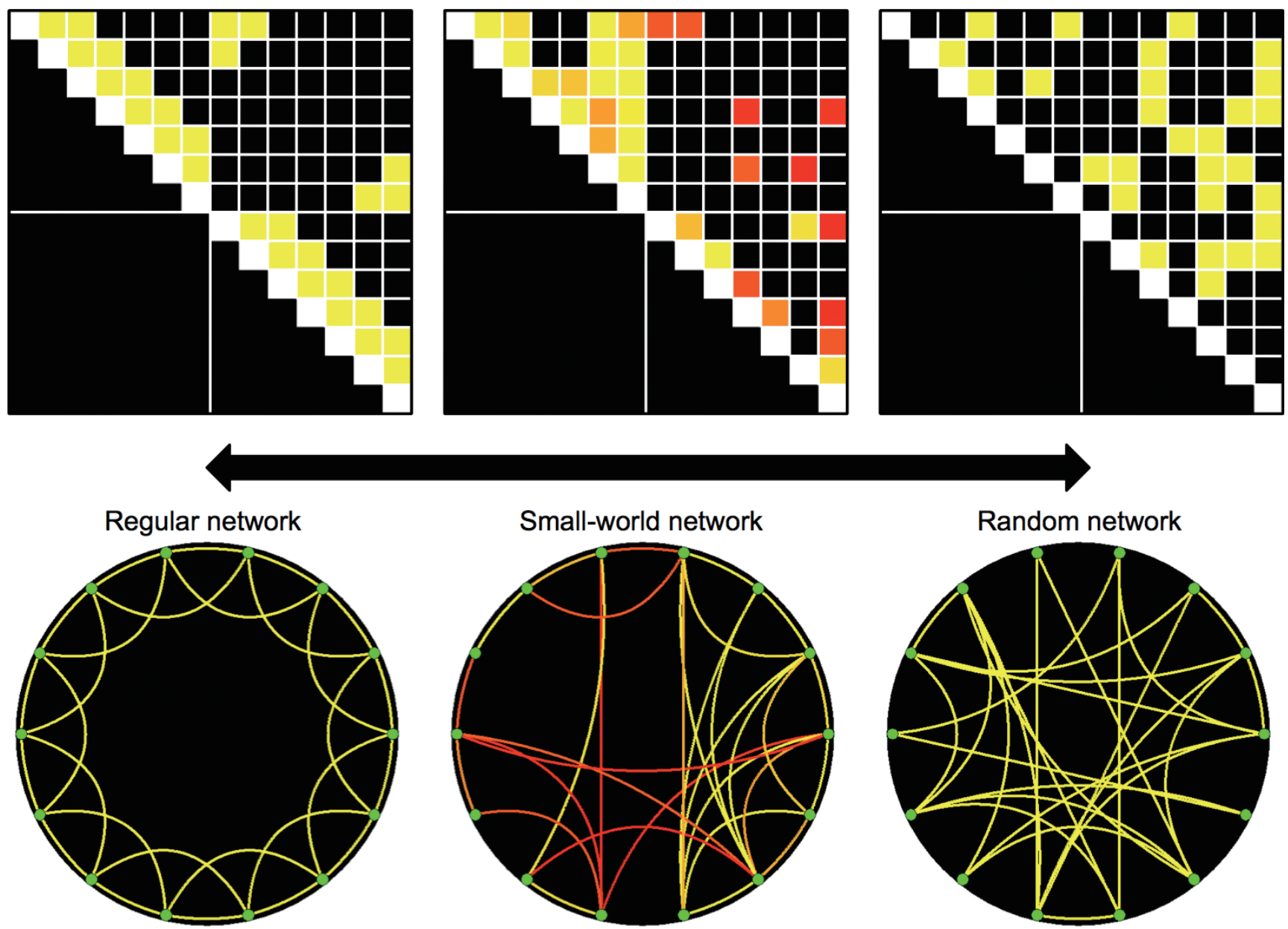

Figure 3. Exemplar networks of regular network (left), small-world network (center) and random network (right). The exemplar small-world network was constructed using the functional connectivity in patients with visual field defect at 1 week after posterior cerebral artery territory infarction $(n=30)$. Colors of connectivity matrix and circular lattice represent statistical significances from one sample $t$ test $(n=30)$ from red $\left(P=10^{-5}\right)$ to yellow colors $\left(P=10^{-10}\right)$. 
the connections are randomly wired, and the wiring probabilities of one specific node to multiple remote nodes are relatively higher than those for adjacent nodes. Thus, remote connections overwhelm neighboring connections, which results in low clustering and small characteristic path length (Figure 3). A smallworld network is characterized by the presence of hubs, which connect remote regions via a small number of steps. Due to the presence of hubs, small-world networks can communicate more efficiently with other nodes, which results in a high mean clustering coefficient and shorter characteristic path lengths. ${ }^{10}$ Realworld networks such as airlines, highways, web hyperlinks, and social networks show these small-worldness characteristics. The small-worldness of brain networks is considered crucial for the maintenance of normal cognitive and behavioral function. It enables the brain to reduce energy consumption and volume, while maximizing the complexity and economic efficiency of communication among neurons. ${ }^{14}$

\section{Visualizing the network}

These network characteristics can be visualized using various methods, such as connectivity matrices, graph theoretical methods, and circular representations (Figure 4). ${ }^{15,16}$ Using structural and functional connectivity data, a connectivity matrix could be constructed among prespecified cortical regions. This gives information about the presence and weights of connections between predetermined nodes in a two-dimensional format. The graph theoretical method consists of a two- or three-dimensional diagram showing a rather limited number of nodes that elucidates the network connectivity characteristics in a graphical way. Finally, the circular representation is another interesting
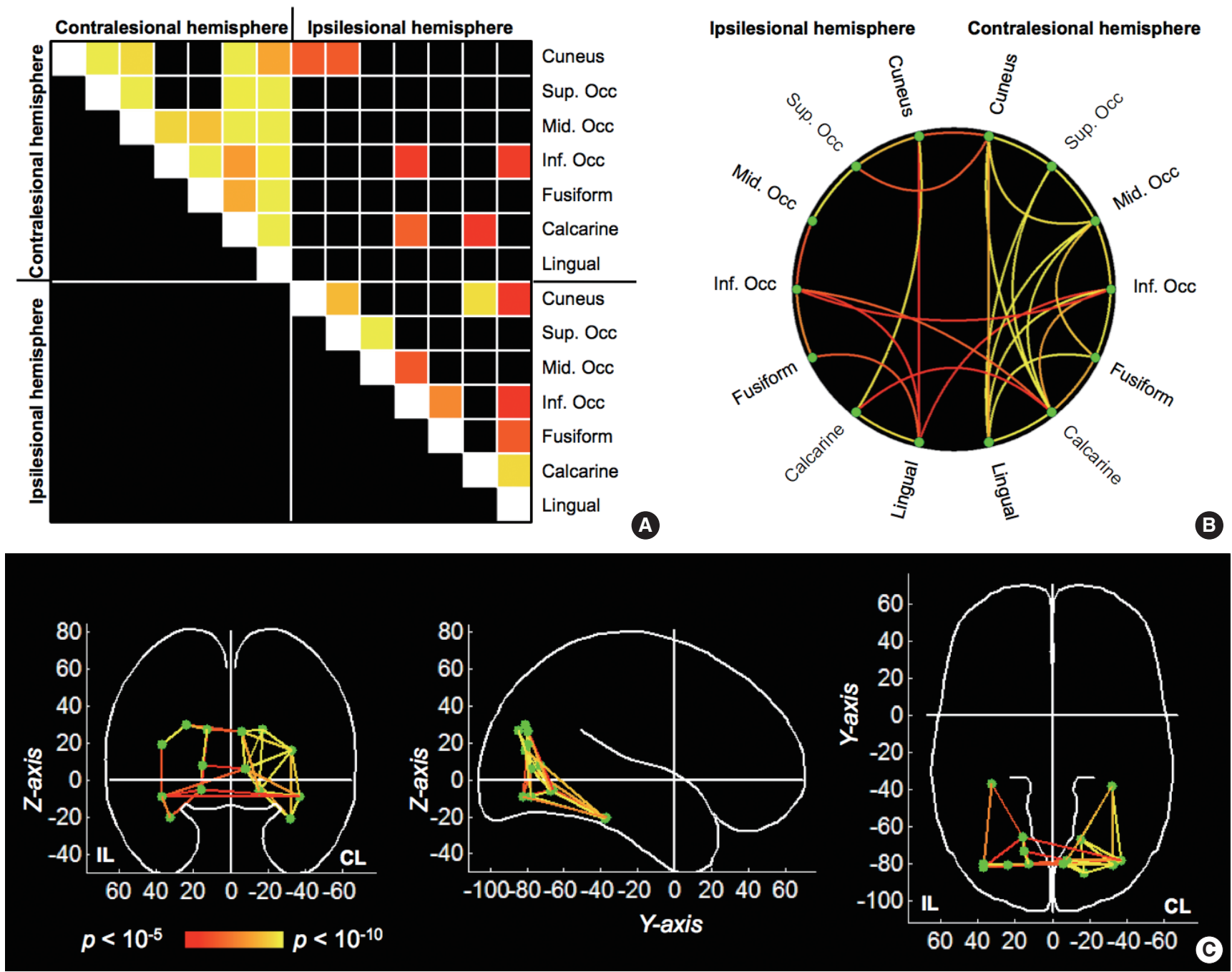

Figure 4. Network visualization by (A) connectivity matrix, (B) circular representation, and (C) graph theoretical methods. The vision-related regions-of-interest were selected as network nodes based on Automated Anatomical Labeling (AAL) atlas. Statistical significance from one sample $t$ test $(n=30)$ was used to represent the network connections between nodes from red $\left(P=10^{-5}\right)$ to yellow colors $\left(P=10^{-10}\right)$. Fisher's z-score was used in statistical tests. IL, ipsilesional hemisphere; $C L$, contralesional hemisphere; Sup, superior; Mid, middle; Inf, inferior; Occ, occipital cortex. 
method by which to characterize and visualize whole-brain connectivities. It was originally proposed as a way to present the associations among large numbers of genetic loci. Currently, it is widely used in various fields, including neuroscience and clinical neurology. ${ }^{6,15,17}$ In this method, each cortical region constitute a ring lattice, and connections inside lattice represent structural and functional connectivities between the cortical regions (Figure 4B).

Describing a detailed methodology for connectome analysis is beyond the scope of this review. In essence, the construction of a connectivity matrix is based on cortical parcellation and tractography. The connectivity matrix holds information regarding the presence and densities of connections within the whole brain, and is the basic unit of connectome analysis. ${ }^{12}$

\section{Connectome perspectives in neurological disorders}

Current evidence for the disruption of structural and functional connectivity in various neurodegenerative dementias have been described thoroughly in recent reviews. ${ }^{10,18}$ Researchers have investigated the associations between more global network architecture parameters (i.e., clustering coefficient, shortest path length, local/global path length) and cognitive functions in each domain in patients with Alzheimer's disease. Patient groups have shown decreased local/global efficiency and increased path length compared to control groups. Based on these findings, a perspective has been developed that views the various neurological disorders as a system dysfunction of widespread and related networks. However, few studies have evaluated these large-scale neural networks or system-wide connectome changes in stroke survivors. ${ }^{19}$

\section{Stroke and connectome}

Why are network and connectome perspectives important for stroke survivors? A recent review cast light on the clinical implications of the connectome approach. ${ }^{20}$ It could be useful to help our understanding of the underlying pathophysiology of stroke symptoms, the mechanisms of cortical reorganization, neural plasticity, and functional recovery after stroke. This approach may enable clinicians to stratify patients according to their chance of recovery after stroke, as well as facilitate the recovery process by individualizing rehabilitation interventions on the basis of understanding the network changes caused by stroke. ${ }^{20}$

\section{Local structural and functional network changes after stroke}

Brain networks consist of functionally segregated groups of neurons and their connections. Thus, focal lesions could affect neighboring neurons and their connections, and might be the first step in understanding the effect of stroke lesions on cerebral network changes after stroke. Early structural changes after stroke in peri-infarct tissue can be summarized as dendritic spine and synaptic loss, which results in the destruction of local networks. Within minutes of ischemia, spinal loss in apical dendrites and glial swelling within neighboring structures are observed. ${ }^{21,22}$ Thereafter, compensatory dendritic spine production occurs in recovering circuits weeks after stroke as lost function is remapped in the peri-infarct zone. ${ }^{23-25}$ These processes have been documented in the neuroimaging research. In task-based fMRI studies, activity of the motor cortex is depressed in the first three days after stroke, then gradually increases in the bilateral primary motor cortex. ${ }^{26}$ The region activated by forming the paretic hand into a fist moves outward to the neighboring cortical areas, beyond the original hand representation, which was destroyed by stroke. In this study, the more severely damaged the corticospinal tract was, the more widely distributed the activated cortical regions were, i.e., remapping of the lost function, even to the contralesional hemispheres.

\section{Remote structural and functional changes after stroke}

Beyond local network changes, stroke lesions can influence remote sites in various ways. This can be understood from the perspective of functional segregation of neural networks. ${ }^{10}$ Structural and functional connectivity might be the basis of understanding these remote changes after stroke. Even small subcortical lacunes in the genu portion of the internal capsule could result in focal cortical thinning in the ipsilateral frontal cortex through destruction of ipsilateral anterior thalamic projections. ${ }^{27,28}$ Furthermore, impairments in processing speed may be attributed to lesions of the anterior thalamic projections and forceps minor, as demonstrated by voxel-based lesion mapping, which confirms that these connecting fibers confer the cognitive functions of the frontal lobe. ${ }^{29}$ Similarly, tractography in diffusion tensor images has revealed the importance of the left arcuate fasciculus for the recovery of language function in chronic stroke survivors. ${ }^{30}$ These studies suggest that the identification and quantification of structural connectivity might be helpful for understanding the underlying mechanisms of stroke sequela and for predicting prognosis.

\section{Intra- and inter-hemispheric connectivity and post- stroke recovery}

There are several lines of evidence for the role of transcallosal interactions between the ipsilesional and contralesional hemispheres in the recovery of lost function. The primary motor cortex is under reciprocal functional influence by contralateral 
homologous regions, via transcallosal connections. Furthermore, the primary motor cortex is functionally connected to neighboring ipsilesional supplementary motor and premotor areas. Thus, stroke lesions in the primary motor cortex could disrupt functional connectivities with ipsilesional neighboring regions and the contralesional primary motor cortex. ${ }^{31}$

Restoration of impaired interhemispheric correlations can predict regaining of function after stroke. ${ }^{32-36}$ Interhemispheric connectivities between posterior intraparietal sulci are correlated with the reaction time to visual stimuli, while intrahemispheric functional connectivity is not associated with attention or motor performance. $^{32}$

These remote effects vary according to the stroke stage. In the acute phase of stroke, contralesional activity might be supportive for the recovery of function. In macaques, when the contralesional primary motor cortex was pharmacologically inactivated a week after stroke in the corticospinal tract, the functional recovery of the lost motor function was disturbed. ${ }^{31}$ In contrast, in chronic stroke survivors with persistent hemiparesis, this transcallosal functional connection might suppress the primary motor cortex of the lesioned hemisphere. ${ }^{37}$ Transient downregulation of the contralesional primary motor cortex leads to improved motor function of the paretic hand. ${ }^{38}$ However, contradictory findings have also been reported. Magnetic encephalography and resting-state fMRI studies have found recovery of lost function to be predicted by decreased activity in the contralateral motor area, and by increased functional activity in the ipsilesional supplemental motor area and cerebellum in the acute phase following stroke. ${ }^{39,40}$

These effects also vary by the extent of lesions. As the corticospinal tract damage becomes more severe, fMRI activity increases more extensively and persistently in the contralesional hemisphere in the chronic phase of stroke (after 6-12 months). Interestingly, these functional associations may be mediated only in cases in which the structural connectivity is maintained at least to some degree. ${ }^{41}$

\section{Changes in large-scale neural networks and global network architecture after stroke}

Relative to task-specific fMRI studies, resting-state neural networks and global network architecture have rarely been investigated. Graph theoretical analysis has suggested that the functional connectivities of major hubs of the default mode network decrease following stroke, including the posterior cingulate cortex, medial prefrontal cortex, and left medial temporal lobe.

Global network architecture measures have been investigated using computational modeling of the impact of artificial lesions in the human brain. ${ }^{42}$ The methods of sequential single node deletions and localized area removal were used to explore the global effects of these artificial lesions on network efficiency. The major findings were that the deletion of highly central nodes could greatly disrupt the interactions between nodes in the remaining brain regions. The cortical midline, temporo-parietal junction, and frontal cortical lesions had the largest and most widespread effects on the global efficiency of the network architecture. In contrast, lesions of the primary visual and somatomotor cortex affected the remaining brain regions relatively modestly. ${ }^{42}$ Although the artificial lesions could not incorporate the possible compensatory mechanisms of the human brain, these results provide connectivity perspectives on global effects of localized lesions. Many aspects of this field remain to be explored through further studies, including the possible associations with long-term outcomes.

\section{The connectome and its implications for cognitive and behavioral sequela after stroke}

\section{Network hubs and post-stroke dementia}

Results from the aforementioned studies using artificial lesions are consistent with recent clinical studies that have shown the importance of network hubs. Cumulative lesion mapping has illustrated that patients with post-stroke dementia show more frequent involvement of cortical hub regions of large-scale neural networks, such as default mode and central executive networks, compared to matched controls without dementia. ${ }^{43}$ Stroke lesions situated in critical hubs, including heteromodal association cortices such as the posterior parietal cortex, cause clinically overt post-stroke dementia. ${ }^{43,44}$ These high-degree hubs consist of a rich-club organization, which serves to integrate information and foster efficient global brain communication. ${ }^{45-47}$ Thus, destruction of those cortical hubs might confer an increased risk of cognitive and behavioral sequela after stroke, compared with damage to other conventional nodes in brain.

\section{Changes in large-scale neural networks and post- stroke cognitive decline}

Default mode network activity has been investigated in patients with chronic right hemispheric stroke using resting-state fMRI. ${ }^{19}$ The functional activity of the left anterior cingulate cortex and left precuneus was higher in stroke survivors than in controls, and these increases in resting-state activities were correlated with line-cancellation test scores. In seed-based connectivity analysis, local default mode network connectivity was lower in stroke survivors than in controls, especially in the medial prefrontal cortex, parahippocampus, and posterior parietal 
cortex, which were the regions impaired in patients with poststroke dementia. ${ }^{43}$

In patients with right hemispheric stroke, ${ }^{48}$ poor cognitive recovery was shown to be associated with increased resting-state functional activity in the contralateral hemisphere, including the default mode, frontotemporal, and basal ganglia networks, while the good cognitive recovery group did not show any significant differences from healthy controls. Although it is unclear whether these increased activities in the contralesional hemisphere were a consequence of compensation or maladaptive behavior, the normalization of these bihemispheric interactions in the good cognitive recovery group favors the possibility of a negative influence of these contralesional functional activities after stroke.

Regarding the underlying structural connectivity changes after stroke, ${ }^{49}$ poor cognitive recovery is associated with decreased fractional anisotropy and increased radial diffusivity in multiple white matter tracts, including the anterior thalamic projections, genu of the corpus callosum, forceps minor, and superior and inferior fronto-occipital fasciculi. Furthermore, fractional anisotropy is associated with processing speed and semantic fluency.

\section{Changes in global network architecture and post- stroke cognitive decline}

The global effects of stroke lesions were explored further in a quantitative stroke lesion-dysfunction mapping study. ${ }^{50}$ This study illustrated the relative contribution of each structural connectivity change to cognitive function. The authors proposed a change in connectivity ( $\mathrm{ChaCo}$ ) score to measure quantitatively the structural disconnections caused by stroke lesions in the predetermined gray matter regions. A higher ChaCo score notes more disruption of structural connectivity in a given region. The associations between $\mathrm{ChaCo}$ scores and each cognitive domain of the Montreal Cognitive Assessment (MoCA) were visualized using three-dimensional glass brain templates.

\section{Aphasia, hemianopia, neglect, and the connectome}

Beyond global cognitive function, specific behavioral sequela such as aphasia, hemianopia, and neglect have also been investigated from a network perspective.

\section{Post-stroke aphasia and network analysis}

In post-stroke survivors with aphasia, the left frontoparietal language network was disrupted in resting-state fMRIs acquired 1 and 3 months after stroke onset. ${ }^{51}$ The functional connectivity between the left frontoparietal network and right middle/medial frontal cortex was also lower, and these changes were associated with the impairment of comprehension abilities. In patients with motor aphasia, functional connectivity was enhanced among the Broca's area and adjacent cortical areas. In addition, the connectivity with contralateral brain regions including the middle temporal gyrus, splenium of the corpus callosum, occipital lobe, and cerebellum were also higher.

Recovery from aphasia after stroke may vary according to lesion locations and size. ${ }^{52}$ In this study, one patient showed acute right-hemisphere substitution of language functions, which was confirmed by a task-specific fMRI study. Disturbance of language networks by stoke could be reversed by language rehabilitation: language network activation was restored to a similar level as that seen in controls after 10 weeks of semantic-feature based language rehabilitation. Another study showed that inhibitory repetitive transcranial magnetic stimulation to the contralesional (right) inferior frontal gyrus was effective in assisting recovery from aphasia. Positron emission tomography showed that activity within the left hemisphere improved after repetitive transcranial magnetic stimulation to the right inferior frontal gyrus. Stimulation of the right hemisphere might have suppressed interhemispheric transfer of language function, while promoting intrahemispheric transfer within the lesioned (left) hemisphere, which engendered the return of language centers to the dominant hemisphere.

During the recovery process, some structural connectivity parameters may be predictors of good outcomes. In a longitudinal study with patients with chronic post-stroke aphasia, the right arcuate fasciculus was illustrated to play an important role in the recovery from aphasia after left-hemispheric stroke. ${ }^{53}$ Preservation of global and local structural connectivity and network efficiency of left temporal cortical regions was also identified as a predictor of good recovery after speech therapy in patients with post-stroke aphasia. ${ }^{54}$

\section{Hemianopia}

The mechanisms of recovery from hemianopia after stroke are still unclear, and few relevant studies have been conducted to date. In patients with hemianopia after mild right occipital infarction, resting-state fMRI and multi-channel electroencephalography have shown that newly formed connections were established between the peri-infarct areas and contralesional frontal, central, and parietal cortices. This interhemispheric connectivity was associated with the extent of hemianopia. A multichannel electroencephalography study showed that global network architecture parameters, including clustering coefficients and characteristic path lengths, did not differ between patients and healthy controls. However, local activation patterns in pa- 
tients with hemianopia were enhanced in the ipsilesional temporopolar and orbitofrontal areas and the contralesional associative visual cortex, compared to healthy controls. These studies suggest the possible roles of rewiring within ipsilesional areas and interhemispheric connectivity for recovery from post-stroke hemianopia.

\section{Neglect}

Network changes in patients with neglect seem to be more complicated. The bihemispheric functional correlation between the posterior intraparietal sulcus of the dorsal attention network may be disrupted in acute phases of right hemispheric stroke, eventually normalizing in the chronic phase of stroke. Furthermore, this bihemispheric correlation was negatively correlated with the reaction time for visual attention tasks. However, lesionmapping studies in patients with neglect have revealed that more ventrally located structural lesions in the inferior parietal lobule, temporoparietal junction, and superior temporal gyrus are important for the development of symptoms. ${ }^{55}$ From a network perspective, the frontoparietal network, which consists of the dorsal frontal cortex and posterior parietal cortex, is usually preserved in patients with stroke with neglect symptoms. ${ }^{4}$ Stroke lesions in the ventral attention network may have remote effects on the dorsal attention networks, which may be responsible for clinical neglect symptoms. In turn, dysfunction of the dorsal attention network is associated with the attentional ability of patients with stroke with spatial neglect. ${ }^{56}$

One insightful study has shown associations between structural connectivity and visual neglect symptoms. ${ }^{57}$ In patients with poor recovery from neglect, the ventral and dorsal attention network fibers were weakened compared to individuals the a good-recovery group or those without neglect. A reduction in fractional anisotropy assessed via diffusion tensor imaging was correlated with visuospatial neglect test scores. Regarding functional connectivity changes in patients with neglect, ${ }^{58}$ interhemispheric functional connectivity has been reported to decrease in the dorsal attention network and sensory-motor network.

In addition to deficits in each cognitive domain, specific behavioral sequela after stroke, such as aphasia, hemianopia, and neglect might also be interpreted and understood within the scope of neural connectivity changes. Each symptom is accompanied by changes in general network architectures, ipsilesional local connectivity, as well as remote interhemispheric connectivities. These findings could enrich our knowledge of the underlying mechanisms, namely which connectivity changes cause which specific symptoms, and the dynamic processes during recovery from those symptoms, and finally could help us to establish strategies to overcome the behavioral sequela.

\section{Perspectives}

\section{Clinical implications of the stroke connectome}

As discussed above, network analysis and a system-level approach based on the connectome in stroke patients might have clinical implications for our understanding of the mechanisms underlying symptoms and functional recovery, including recovery from cognitive dysfunction. ${ }^{4,20}$ Beyond the classical lesionmapping approach, multi-network assessments and connectome-based analyses might provide more reasonable explanations for the neurologic deficits and recovery process after stroke. Furthermore, novel therapeutic interventions that disrupt the maladaptive processes and facilitate neural circuit recovery might also be possible, such as modulation of primary motor cortex function by repetitive transcranial magnetic stimulation. ${ }^{38}$

Similarly, network perspectives for stroke symptomatology and recovery mechanisms might open a new era of research and practice in clinical neurology and neurorehabilitation. Such perspectives might also be used to stratify patients' possibilities of recovery, and to select proper, tailored rehabilitation programs that provide optimized individual therapies to strengthen the recovery of impaired network organization. In addition, monitoring the whole recovery process to ensure implementation of the proper rehabilitation program and tracking transitional states within the dynamic temporal course of neuroplasticity might be also possible.

\section{Limitations and technical pitfalls}

There are several limitations to the approaches detailed in this review. First, the accuracy and reproducibility of reconstructions of structural and functional connectivities might be a problem. Because of the various cortical parcellation schemes and the resolution issues of diffusion tensor imaging, the results of connectivity analyses might vary, even for the same connectome-analysis method. Technical improvements should lead to better fiber tracking; however, as the development of diffusion tensor or spectral imaging progresses, the computational burden simultaneously increases. More robust mathematical and computational methodologies are needed to overcome this computational burden.

There is a lack of confirmatory evidence for the human pathologic correlates of neuroimaging findings such as fractional anisotropy, and radial or axial diffusivities. Stroke survivors of advanced age usually have mixed pathology, including amyloidopathy, tauopathy, and synucleinopathy, as well as vascular atherosclerosis. Resting-state fMRI cannot be appropriately interpreted in cases with moderate to severe intracranial stenosis. Multiple destructive lesions and morphological variances, including old 
territorial infarction, multiple lacunes, and severe cerebral atrophy might distort the results of standardized analysis protocols. Finally, the structural connectome approach might not fully account for the extrasynaptic communications and interactions among neurons. ${ }^{59}$ These technical pitfalls should be considered prior to adopting the recent connectome research findings into clinical practice.

\section{Conclusion}

Major cortical hub regions, large-scale neural network integrities, inter-hemispheric connectivity, and aspects of general network architecture might be important for maintaining normal cognitive function after stroke. The 4 Rs have been mentioned as implications of the connectome for understanding dynamic processes in our brain, namely "regeneration," "reconnection," "reweighting," and "rewiring." 59 Our understanding of various stroke symptoms and their associated recovery processes might be interpreted similarly.

In conclusion, understanding the stroke connectome and dynamic network changes throughout the acute and chronic phases of stroke give us the opportunity to monitor the recovery process, provide individualized and tailored rehabilitation, and offer novel neuromodulatory therapies. Therapeutic interventions to overcome the network disruption and to improve global and local efficiencies might also follow.

\section{Acknowledgements}

We appreciate Yong-Hwan Kim's assistance in preparing the figures.

\section{References}

1. Sporns O, Tononi G, Kotter R. The human connectome: A structural description of the human brain. PLoS Comput Biol 2005; $1: \mathrm{e} 42$.

2. Sporns O. Discovering the Human Connectome. Cambridge, MA: The MIT Press, 2012.

3. Monakow C. Die Lokalisation im Grosshirn und der Abbau der Funktion durch kortikale Herde. Wiesbaden: Bergmann JF, 1914.

4. Carter AR, Shulman GL, Corbetta M. Why use a connectivitybased approach to study stroke and recovery of function? Neuroimage 2012;62:2271-2280.

5. Euler L. Solutio problematis ad geometriam situs pertinentis. Novi Commentarii Academiae Scientarum Imperialis Petropolitanae 1741;8:128-140.
6. Petrella JR, Doraiswamy PM. From the bridges of Konigsberg to the fields of Alzheimer: Connecting the dots. Neurology 2013; 80:1-3.

7. Greicius MD, Supekar K, Menon V, Dougherty RF. Restingstate functional connectivity reflects structural connectivity in the default mode network. Cereb Cortex 2009;19:72-78.

8. Huettel S, Song A, McCarthy G. BOLD fMRI: Origins and Properties. Functional magnetic resonance imaging. MA, USA: Sinauer Associates, Inc., 2009; 193-242.

9. Grefkes C, Fink GR. Reorganization of cerebral networks after stroke: New insights from neuroimaging with connectivity approaches. Brain 2011;134:1264-1276.

10. Pievani M, de Haan W, Wu T, Seeley WW, Frisoni GB. Functional network disruption in the degenerative dementias. Lancet Neurol 2011;10:829-843.

11. Friston KJ. Functional and effective connectivity in neuroimaging: A synthesis. Hum Brain Mapp 1994;2:56-78.

12. Rubinov M, Sporns O. Complex network measures of brain connectivity: Uses and interpretations. Neuroimage 2010;52: 1059-1069.

13. Watts DJ, Strogatz SH. Collective dynamics of 'small-world' networks. Nature 1998;393:440-442.

14. Sporns O. The Brain's Small World. Networks of the Brain. Massachusetts: MIT press, 2011;125.

15. Irimia A, Chambers MC, Torgerson CM, Van Horn JD. Circular representation of human cortical networks for subject and population-level connectomic visualization. Neuroimage 2012; 60:1340-1351.

16. Craddock RC, Jbabdi S, Yan C-G, Vogelstein JT, Castellanos FX, Di Martino A, et al. Imaging human connectomes at the macroscale. Nat Methods 2013;10:524-539.

17. Irimia A, Chambers MC, Torgerson CM, Filippou M, Hovda $\mathrm{Da}$, Alger JR, et al. Patient-tailored connectomics visualization for the assessment of white matter atrophy in traumatic brain injury. Front Neurol 2012;3:10.

18. Filippi M, Agosta F. Structural and functional network connectivity breakdown in Alzheimer's disease studied with magnetic resonance imaging techniques. J Alzheimer's Dis 2011;24: 455-474.

19. Dacosta-Aguayo R, Graña M, Iturria Medina Y, Fernández-Andújar M, López-Cancio E, Cáceres C, et al. Impairment of functional integration of the default mode network correlates with cognitive outcome at three months after stroke. Hum Brain Mapp 2015;36:577-590.

20. Grefkes C, Fink GR. Connectivity-based approaches in stroke and recovery of function. Lancet Neurol 2014;13:206-216.

21. Murphy TH, Li P, Betts K, Liu R. Two-photon imaging of stroke onset in vivo reveals that NMDA-receptor independent 
ischemic depolarization is the major cause of rapid reversible damage to dendrites and spines. J Neurosci 2008;28:1756-1772.

22. Risher WC, Croom D, Kirov SA. Persistent astroglial swelling accompanies rapid reversible dendritic injury during stroke-induced spreading depolarizations. Glia 2012;60:1709-1720.

23. Brown CE, Aminoltejari K, Erb H, Winship IR, Murphy TH. In vivo voltage-sensitive dye imaging in adult mice reveals that somatosensory maps lost to stroke are replaced over weeks by new structural and functional circuits with prolonged modes of activation within both the peri-infarct zone and distant sites. JNeurosci 2009;29:1719-1734.

24. Mostany R, Chowdhury TG, Johnston DG, Portonovo SA, Carmichael ST, Portera-Cailliau C. Local hemodynamics dictate long-term dendritic plasticity in peri-infarct cortex. J Neurosci 2010;30:14116-14126.

25. Johnston DG, Denizet M, Mostany R, Portera-Cailliau C. Chronic in vivo imaging shows no evidence of dendritic plasticity or functional remapping in the contralesional cortex after stroke. Cereb Cortex 2013;23:751-762.

26. Rehme AK, Fink GR, von Cramon DY, Grefkes C. The role of the contralesional motor cortex for motor recovery in the early days after stroke assessed with longitudinal FMRI. Cereb Cortex 2011;21:756-768.

27. Duering M, Righart R, Wollenweber FA, Zietemann V, Gesierich B, Dichgans M. Acute infarcts cause focal thinning in remote cortex via degeneration of connecting fiber tracts. Neurology 2015;84:1685-1692.

28. Duering M, Righart R, Csanadi E, Jouvent E, Herve D, Chabriat $\mathrm{H}$, et al. Incident subcortical infarcts induce focal thinning in connected cortical regions. Neurology 2012;79:2025-2028.

29. Duering M, Zieren N, Herve D, Jouvent E, Reyes S, Peters N, et al. Strategic role of frontal white matter tracts in vascular cognitive impairment: a voxel-based lesion-symptom mapping study in CADASIL. Brain 2011;134:2366-2375.

30. Marchina S, Zhu LL, Norton A, Zipse L, Wan CY, Schlaug G. Impairment of speech production predicted by lesion load of the left arcuate fasciculus. Stroke 2011;42:2251-2256.

31. Zaaimi B, Edgley SA, Soteropoulos DS, Baker SN. Changes in descending motor pathway connectivity after corticospinal tract lesion in macaque monkey. Brain 2012;135:2277-2289.

32. Carter AR, Astafiev SV, Lang CE, Connor LT, Rengachary J, Strube MJ, et al. Resting interhemispheric functional magnetic resonance imaging connectivity predicts performance after stroke. Ann Neurol 2010;67:365-375.

33. Dubovik S, Pignat JM, Ptak R, Aboulafia T, Allet L, Gillabert $\mathrm{N}$, et al. The behavioral significance of coherent resting-state oscillations after stroke. Neuroimage 2012;61:249-257.

34. Wang L, Guo X, Sun J, Jin Z, Tong S. Cortical networks of hemianopia stroke patients: a graph theoretical analysis of EEG signals at resting state. Conf Proc IEEE Eng Med Biol Soc 2012; 2012:49-52.

35. Liu J, Qin W, Zhang J, Zhang X, Yu C. Enhanced interhemispheric functional connectivity compensates for anatomical connection damages in subcortical stroke. Stroke 2015;46: 1045-1052.

36. Crofts JJ, Higham DJ, Bosnell R, Jbabdi S, Matthews PM, Behrens TEJ, et al. Network analysis detects changes in the contralesional hemisphere following stroke. Neuroimage 2011;54:161169.

37. Grefkes C, Eickhoff SB, Nowak DA, Dafotakis M, Fink GR. Dynamic intra- and interhemispheric interactions during unilateral and bilateral hand movements assessed with fMRI and DCM. Neuroimage 2008;41:1382-1394.

38. Takeuchi N, Chuma T, Matsuo Y, Watanabe I, Ikoma K. Repetitive transcranial magnetic stimulation of contralesional primary motor cortex improves hand function after stroke. Stroke 2005;36:2681-2686.

39. Park CH, Chang WH, Ohn SH, Kim ST, Bang OY, Pascual-Leone A, et al. Longitudinal changes of resting-state functional connectivity during motor recovery after stroke. Stroke 2011; 42:1357-1362.

40. Westlake KP, Hinkley LB, Bucci M, Guggisberg AG, Byl N, Findlay AM, et al. Resting state alpha-band functional connectivity and recovery after stroke. Exp Neurol 2012;237:160-169.

41. Carter AR, Patel KR, Astafiev SV, Snyder AZ, Rengachary J, Strube MJ, et al. Upstream dysfunction of somatomotor functional connectivity after corticospinal damage in stroke. Neurorehabil Neural Repair 2012;26:7-19.

42. Alstott J, Breakspear M, Hagmann P, Cammoun L, Sporns O. Modeling the impact of lesions in the human brain. PLoS Comput Biol 2009; 5:e1000408.

43. Lim JS, Kim N, Jang MU, Han MK, Kim SY, Baek MJ, et al. Cortical hubs and subcortical cholinergic pathways as neural substrates of poststroke dementia. Stroke 2014;45:1069-1076.

44. Sporns O, Honey CJ, Kotter R. Identification and classification of hubs in brain networks. PLoS One 2007;2:e1049.

45. van den Heuvel MP, Sporns O. Rich-club organization of the human connectome. J Neurosci 2011;31:15775-15786.

46. van den Heuvel MP, Stam CJ, Kahn RS, Hulshoff Pol HE. Efficiency of functional brain networks and intellectual performance. J Neurosci 2009;29:7619-7624.

47. Bullmore E, Sporns O. The economy of brain network organization. Nat Rev Neurosci 2012;13:336-349.

48. Dacosta-Aguayo R, Graña M, Savio A, Fernández-Andújar M, Millán M, López-Cancio E, et al. Prognostic value of changes in resting-state functional connectivity patterns in cognitive re- 
covery after stroke: A $3 \mathrm{~T}$ fMRI pilot study. Hum Brain Mapp 2014;35:3819-3831.

49. Dacosta-Aguayo R, Graña M, Fernández-Andújar M, LópezCancio E, Cáceres C, Bargalló N, et al. Structural integrity of the contralesional hemisphere predicts cognitive impairment in ischemic stroke at three months. PLoS One 2014;9:e86119.

50. Kuceyeski A, Navi BB, Kamel H, Relkin N, Villanueva M, Raj A, et al. Exploring the brain's structural connectome: A quantitative stroke lesion-dysfunction mapping study. Hum Brain Mapp 2015: $36: 2147-2160$.

51. Zhu D, Chang J, Freeman S, Tan Z, Xiao J, Gao Y, et al. Changes of functional connectivity in the left frontoparietal network following aphasic stroke. Front Behav Neurosci 2014;8:167.

52. Jarso S, Li M, Faria A, Davis C, Leigh R, Sebastian R, et al. Distinct mechanisms and timing of language recovery after stroke. Cogn Neuropsychol 2013;30:454-475.

53. Forkel SJ, Thiebaut de Schotten M, Dell'Acqua F, Kalra L, Murphy DG, Williams SC, et al. Anatomical predictors of aphasia recovery: A tractography study of bilateral perisylvian language networks. Brain 2014;137:2027-2039.

54. Bonilha L, Gleichgerrcht E, Nesland T, Rorden C, Fridriksson
J. Success of anomia treatment in aphasia is associated with preserved architecture of global and left temporal lobe structural networks. Neurorehabil Neural Repair 2015. doi:10.1177/ 1545968315593808 .

55. Ptak R, Schnider A. The attention network of the human brain: relating structural damage associated with spatial neglect to functional imaging correlates of spatial attention. Neuropsychologia 2011;49:3063-3070.

56. Ptak R, Schnider A. The dorsal attention network mediates orienting toward behaviorally relevant stimuli in spatial neglect. J Neurosci 2010;30:12557-12565.

57. Umarova RM, Reisert M, Beier TU, Kiselev VG, Kloppel S, Kaller CP, et al. Attention-network specific alterations of structural connectivity in the undamaged white matter in acute neglect. Hum Brain Mapp 2014;35:4678-4692.

58. Baldassarre A, Ramsey L, Hacker CL, Callejas A, Astafiev SV, Metcalf NV, et al. Large-scale changes in network interactions as a physiological signature of spatial neglect. Brain 2014;137: 3267-3283.

59. Seung S. Connectome: How the Brain's Wiring Makes Us Who We Are. New York, USA: Mariner Books, 2013. 